\title{
THE STRUCTURE SHEAF OF AN INCIDENCE ALGEBRA
}

\author{
BETH GOLDSTON BARNWELL and A. C. MEWBORN \\ (Received 13 January 1977, revised 26 July 1977) \\ Communicated by $\mathbf{H}$. Lausch
}

\begin{abstract}
Let $R$ be the incidence algebra of a finite partially ordered set $T$ over a commutative noetherian ring $A$. Then the spectrum of $R$ is homeomorphic to the product (Spec $A) \times T$, where $\operatorname{Spec} A$ has the usual Zariski topology and $T$ has the order topology. An explicit construction is given for the structure sheaf of $R$ over its spectrum.
\end{abstract}

Subject classification (Amer. Math. Soc. (MOS) 1970): 16 A 08, 16 A 52.

\section{Introduction}

In Goldston and Mewborn (1975) we define $\operatorname{Spec} R$, the spectrum of an arbitrary left noetherian ring $R$, to be the set of isomorphism classes of indecomposable injective left $R$-modules, and define a topology on $\operatorname{Spec} R$ which reduces to the usual Zariski topology when $R$ is commutative. In addition, we define a sheaf of rings over $\operatorname{Spec} R$ which reduces to the usual structure sheaf when $R$ is commutative. In this paper we show that the spectrum of the incidence algebra $R$ of a finite partially ordered set $T$ over a commutative noetherian ring $A$ is homeomorphic to $(\operatorname{Spec} A) \times T$ with the product topology, where $\operatorname{Spec} A$ has the Zariski topology and $T$ has the order topology. In addition, we explicitly compute the rings of sections over arbitrary open subsets of $\operatorname{Spec} R$.

For a detailed description of the spectrum of a left noetherian ring and the structure sheaf over its spectrum, see Goldston and Mewborn (1977). For standard notions on torsion theories and quotient rings, see Stenström (1971), and for the notions of Krull dimension and critical modules, refer to Gordon and Robson (1973).

The isomorphism class of a module $M$ is denoted by $[M]$, the injective hull of $M$ by $E(M)$. Let $R$ be a noetherian ring, $X=\operatorname{Spec} R$. For each $x \in X$ we fix a representative $V_{x}$ from the isomorphism class $x$. Thus, $x=\left[V_{x}\right]$. If $U \subseteq X$, we denote by $V_{U}$ the direct sum $\amalg\left\{V_{x} \mid x \in U\right\}$. Since $R$ is noetherian, $V_{U}$ is injective. $T_{U}(R)$ denotes the torsion ideal of $R$ in the torsion theory congenerated by $V_{U}$; $E_{U}$ denotes the $R$-endomorphism ring of $V_{U}$ and $R_{U}$ denotes the biendomorphism ring of $V_{U}$. If $U$ is a nonempty open subset of $X, R_{U}$ is the ring of sections over $U$ in the structure sheaf of rings over $X$. 
Throughout, $A$ denotes a commutative noetherian ring with unity, $T$ a finite partially ordered set, $R$ the incidence algebra of $T$ over $A$. All $R$-modules are left modules, and all $R$ - and $A$-modules are unital. Endomorphisms of $R$-modules are written on the right, and a left $R$-module is regarded as a right module over its endomorphism ring. If $A$ is a commutative noetherian ring, there is a one-toone correspondence between prime ideals of $A$ and isomorphism classes of indecomposable injective $A$-modules given by $P \leftrightarrow[E(A / P)]$. Therefore, to simplify notation in this case, we often refer, as is custom, to the point in $\operatorname{Spec} A$ as $P$ rather than $[E(A / P)]$.

\section{A special case}

We first summarize without proofs the results in the special case where $A$ is a field $k$. This case should provide some insight into the general situation.

Let $T$ be a finite partially ordered set, $k$ a field, $R$ the incidence algebra of $T$ over $k$. Then $R$ has $k$-basis $\left\{e_{s, t} \mid s, t \in T, s \leqslant t\right\}$, with multiplication defined by $e_{s t} e_{w u}=\delta_{t, w} e_{s, u}$, where $\delta_{t, w}$ is the Kronecker delta. Denote by $V$ the $k$-vector space with basis $\left\{v_{t} \mid t \in T\right\}$. Then $V$ is a left $R$-module under the module composition defined by $e_{s, t} v_{v o}=\delta_{l, v} v_{g}$.

Let $n$ be the number of elements in $T$. If we choose a linear ordering of $T$ compatible with the partial ordering, then we can identify $R$ with a subalgebra of the algebra of $n \times n$ upper triangular matrices in such a way that each $e_{s, t}$ is identified with one of the canonical matrix units. We can also identify $V$ with the set of $n$-dimensional column vectors in such a way that each $v_{t}$ is identified with a canonical basis vector and such that the module action of $R$ on $V$ is given by matrix multiplication.

For each $t \in T$, let $M_{l}$ be the $k$-subspace of $V$ spanned by $\left\{v_{s} \mid s \ngtr t\right\}$, and let $N_{t}$ be the $k$-subspace of $V$ spanned by $\left\{v_{s} \mid s \neq t\right\}$. Then $M_{t}$ and $N_{t}$ are $R$-submodules of $V$ and $M_{t} / N_{t}$ is a one-dimensional simple $R$-module. In fact, every simple $R$-module is isomorphic to a module of this form. The module $V / N_{l}$ is an injective envelope of $M_{t} / N_{t}$. Furthermore, if $s, t \in T$, then $\operatorname{Hom}_{R}\left(V / N_{s}, V / N_{t}\right) \neq 0$ if and only if $s \leqslant t$, and in this case $\operatorname{Hom}_{R}\left(V / N_{s}, V / N_{\ell}\right)$ has dimension 1 over the field $k$. Thus Spec $R$ is homeomorphic to $T$ with the order topology.

Let $U$ be any open subset of $T$. We wish to describe the ring $R_{U}$ of sections over $U$. First we note that $T_{U}(R)$, which is the kernel of the restriction map $R \rightarrow R_{U}$, is the $k$-subspace of $R$ spanned by $\left\{e_{s, u} \mid s \neq t\right.$, all $\left.t \in U\right\}$. Since $R / T_{U}(R)$ is isomorphic to the incidence algebra of $T^{\prime}$ over $k$, where $T^{\prime}=\{s \in T \mid s \geqslant t$, some $t \in U\}$, we can reduce to the case where $T_{U}(R)=(0)$, that is, where $U$ is dense in $T$. We now make this assumption. Let $Y$ be the set of minimal elements of $T$ and note that the restriction maps $R \rightarrow R_{U}$ and $R_{U} \rightarrow R_{X}$ are injective. We first describe $R_{Y}$ and then characterize $R_{U}$ as a subring of $R_{Y}$. 
For the open set $Y, V_{Y}=\amalg_{y \in Y}\left(V / N_{y}\right)$ and there are no nonzero $R$-module maps from $V / N_{y}$ to $V / N_{z}, y, z \in Y, y \neq z$. Thus, $\operatorname{End}_{R} V_{Y}=\prod_{y \in Y}\left(\operatorname{End}_{R} V / N_{y}\right)$, and Biend $_{R} V_{Y}=\prod_{y \in Y}\left(\operatorname{Biend}_{R} V / N_{y}\right)$. Now End $R / N_{y} \cong k$, all $y \in Y$ and so Biend ${ }_{R}$ $V / N_{y}$ is the full ring of $k$-linear transformations of $V / N_{y}$. Therefore, $R_{Y}$ is a product of full linear rings.

We now return to the characterization of $R_{U}$. We must characterize those elements $\left(q_{y}\right) \in \Pi_{y_{\in} Y}\left(\right.$ Biend $\left._{R} V / N_{y}\right)$ such that $\left(q_{y}\right)$ is the restriction to $V_{Y}$ of an element of Biend ${ }_{R} V_{U}$. For each $y \in Y, V / N_{y}$ has $k$-basis $\left\{v_{t}+N_{y} \mid t \geqslant y\right\}$. To each linear transformation $q_{y}$ of $V / N_{\nu}$ to itself, we can associate the "matrix" $\left[\alpha(y)_{s, t}\right]$ of $q_{y}$ in this basis: $q_{y}\left(v_{t}+N_{y}\right)=\sum_{s \geqslant \nu} \alpha(y)_{s, t}\left(v_{s}+N_{y}\right), t \geqslant y$.

With these notations, the ring $R_{U}$ of sections over $U$ may be identified with the subring of $\prod_{y \in Y}\left(\operatorname{Biend}_{R} V / N_{y}\right)$ consisting of those elements $\left(q_{y}\right)$ such that the associated matrices $\left[\alpha(y)_{s, t}\right]$ satisfy the following two conditions:

(1) For each $y \in Y$ and $s, t \geqslant y, \alpha(y)_{s, t}=0$ if there exists $s^{\prime} \in U$ such that $y \leqslant s^{\prime} \leqslant s$, $s^{\prime} \$ t$.

(2) If $y, z \in Y$ and $s, t \in T$ such that $s, t \geqslant y$ and $s, t \geqslant z$, then $\alpha(y)_{s, t}=\alpha(z)_{s, t}$ if there exists $s^{\prime} \in U$ such that $s, t \geqslant s^{\prime}$ and $s^{\prime} \geqslant y, z$.

When the open set $U$ has a unique minimal element $y$, the description of the ring $R_{U}$ is simpler, since condition (2) above has no content in this case. We define a binary relation $\sigma$ on $T$ as follows: if $s, t \in T$ then $s \sigma t$ provided that for each $t^{\prime} \in U, t^{\prime} \leqslant s$ inplies $t^{\prime} \leqslant t$. Then $\sigma$ is a transitive relation on $T$ which extends the partial order relation. It follows immediately that $R_{U}$ can be identified with the incidence algebra of $T$ with the relation $\sigma$.

For example, let $T=\{1,2,3,4\}$ be the partially ordered set defined by: $1 \leqslant 2 \leqslant 4$, $1 \leqslant 3 \leqslant 4$. $R$ is the ring of $4 \times 4$ triangular matrices of the form

$$
\left[\begin{array}{llll}
* & * & * & * \\
0 & * & 0 & * \\
0 & 0 & * & * \\
0 & 0 & 0 & *
\end{array}\right] .
$$

Let $U=\{1,2\}$. Then the relation $\sigma$ determined by $U$ is obtained from the partial order by the addition of the pairs $(4,2),(3,1)$ and $(3,2)$. Hence, $R_{U}$ can be identified with the ring of $4 \times 4$ matrices of the form

$$
\left[\begin{array}{llll}
* & * & * & * \\
0 & * & 0 & * \\
* & * & * & * \\
0 & * & 0 & *
\end{array}\right] .
$$




\section{General case}

Let $T$ be a finite partially ordered set and $A$ a commutative noetherian ring. The incidence algebra $R$ of $T$ over $A$ is the free $A$-module with $A$-basis $\left\{e_{s, t} \mid s, t \in T\right.$, $s \leqslant t\}$, with multiplication defined by $e_{g, t} e_{w, u}=\delta_{l w} e_{s, w}$, where $\delta_{t, w}$ is the Kronecker delta.

Let $n$ be the number of elements of $T$ and choose a linear ordering of $T$ compatible with partial ordering. Then we can identify $R$ with a subring of the ring of $n \times n$ upper triangular matrices over $A$ in such a way that $e_{s, t}$ is identified with one of the canonical matrix units.

If $L$ is an $A$-module, define $V(L)$ to be the set of functions from $T$ into $L$. Let $\ell v_{t}$ denote the function $v \in V(L)$ such that $v(t)=\ell$ and $v(s)=0, s \neq t$. Let $L v_{t}=\{v \in V(L) \mid v(s)=0, s \neq t\}$. Note that $V(L)$ is a left $R$-module under the module composition $e_{s, t} \ell v_{w}=\delta_{t, w} \ell v_{s}$. If we identify $V(L)$ with the set of $n$-dimensional column vectors with entries in $L$, the module action of $R$ on $V(L)$ is given by matrix multiplication. For each $t \in T$, we also define

and

$$
M_{l}(L)=\{v \in V(L) \mid v(s)=0 \text { if } s>t\}
$$

$$
N_{b}(L)=\{v \in V(L) \mid v(s)=0 \text { if } s \geqslant t\} .
$$

Note that $M_{l}(L)$ and $N_{l}(L)$ are $R$-submodules of $V(L)$ and $M_{l}(L) / N_{l}(L)$ is $A$-isomorphic to $L$. To simplify notation, we let $K_{l}(L)$ denote $M_{l}(L) / N_{l}(L)$.

An $R$-module $M$ is critical if the Krull dimension of every proper factor of $M$ is less than the Krull dimension of $M$ (see Gordon and Robson, 1973). If $P \in \operatorname{Spec} A$ then $K_{t}(A / P)$ is a critical $R$-module for each $t \in T$. Two critical modules are equivalent if they have isomorphic injective envelopes.

Proposition 3.1. The set $\left\{K_{l}(A / P) \mid t \in T, P \in \operatorname{Spec} A\right\}$ is a complete set of equivalence class representatives of critical $R$-modules. The $R$-module $V(E(A / P)) / N_{l}(E(A / P))$ is an injective envelope of $K_{\iota}(A / P)$.

Proof. First note that if $R$ is critical as a module over itself, then $R \cong A$ and $T$ has only one element. In this case, the result is well known. Now assume $C$ is a nonzero cyclic critical $R$-module; $C \cong R / I$, where $I$ is a proper left ideal of $R$. Choose $t \in T$ such that $e_{t, t} \notin I$. Then $R e_{t, t} /\left(R e_{t, t} \cap I\right)$ is isomorphic to a submodule of $C$, and we may assume $C$ is of this form. Write $R e_{t, t} \cap I=\sum_{s \leqslant t}\left(I_{s} e_{s t}\right)$, where $I_{s}$ is an ideal in $A$ for all $s \leqslant t$ in $T$ and $I_{u} \subseteq I_{s}$ if $s \leqslant u$ in $T$. Choose $u \in T$ minimal such that $u \leqslant t$ and $A / I_{u} \neq(0)$. Then $K_{u}\left(A / I_{u}\right) \cong\left(\sum_{s \leqslant u} A e_{s, t}\right) /\left(\sum_{s \leqslant u} I_{s} e_{s, t}\right)$, and this is isomorphic to a submodule of $C$. Thus we can reduce to the case $C \cong K_{u}(A / I)$ where $I$ is an ideal of $A$. Since $C$ is uniform as an $R$-module, $A / I$ is a uniform $A$-module. Thus $A / I \subseteq E(A / P)$ for some prime ideal $P$ of $A$. Furthermore, there is an injection $A / P \rightarrow A / I$. This induces an injection of the $R$-module $K_{u}(A / P)$ into $C$. Thus $C$ is equivalent to a critical module of the required form. 
Let $V_{P, t}=V\left(E(A / P) / N_{t}(E(A / P))\right.$, where $t \in T, P \in \operatorname{Spec} A$; and note that every nonzero submodule of $V_{P, l}$ must have nonzero intersection with $K_{l}(A / P)$. Thus $V_{P, t}$ is indecomposable. We must show that $V_{P, t}$ is injective as an $R$-module.

If $V_{P, l}$ is not injective, there is a proper left ideal $I$ of $R$ and an $R$-map $f: I \rightarrow V_{P, l}$ which has no extension as an $R$-module map to a left ideal properly containing $I$. Choose $s \in T$ such that $e_{s, s} \notin I$. First suppose that $\operatorname{Re}_{s, s} \cap I \subseteq \operatorname{Ker} f$. Then define $g: R e_{s, s}+I \rightarrow V_{P, l}$ by $g: r e_{s, s}+a \rightarrow a f, a \in I, r \in R$. If $r e_{s, s}+a=0$,

$$
a=-r e_{s, s} \in R e_{s, s} \cap I \subseteq \operatorname{Ker} f,
$$

and $a f=0$. Hence $g$ is a well-defined $R$-map properly extending $f$, a contradiction.

Therefore, $R e_{s, s} \cap I \ddagger \operatorname{ker} f$. Since $V_{P, l}$ is uniform, we may choose $r_{0} \in R$ such that $r_{0} e_{s, s} \in I$ and $0 \neq\left(r_{0} e_{s, s}\right) f \in K_{t}(A / P)$. Thus $\left(r_{0} e_{s, s}\right) f=\alpha v_{t}+N_{t}(E(A / P)), \alpha \in A / P$. But then also $\left(e_{t, l} r_{0} e_{s, s}\right) f=\alpha v_{t}+N_{l}(E(A / P))$. Write $e_{t, l} r_{0} e_{s, s}=a_{t, s} e_{t, s}, a_{t, s} \in A$, and let $J=\left\{a \in A \mid a e_{t, s} \in I\right\}$. Then $J$ is an ideal of $A$ and the composition $g: J \rightarrow E(A / P)$ given by

$$
j \mapsto j e_{t, 8} \mapsto\left(j e_{t, s}\right) f=\beta v_{l}+N_{l}(E(A / P)) \mapsto \beta, \quad \text { where } j \in J, \beta \in E(A / P),
$$

is a nonzero $A$-map. Since $J \subseteq A, g$ extends to a map $g^{\sharp}: A \rightarrow E(A / P)$. Let $c=1 g^{\#}$, and define $h: I+R e_{s, s} \rightarrow V_{P, l}$ by $h: a+r e_{s, s} \mapsto a f+c r v_{s}+N_{t}(E(A / P)), a \in I, r \in R$. Suppose that $a=-r e_{s, s} \in I \cap R e_{s, s}$, and write $r=\sum_{u \leq v} a_{u, v} e_{u, v}, a_{u, v} \in A$. Then $r e_{s, s}=\sum_{u \leq s} a_{u, s} e_{u, s}, r v_{s}+N_{t}(E(A / P))=\sum_{u \leq s}\left[a_{u, s} v_{u}+N_{b}(E(A / P))\right]$. Now

$$
e_{u, u} r e_{s, s}=a_{u, s} e_{u, s}
$$

for all $u \leqslant s$ and so $a_{u, s} e_{u, s} \in I$. Further, if $a_{u s,} \neq 0$,

$$
\left(e_{u, u} a_{u s} e_{u, s}\right) f=\left(a_{u, s} e_{u, s}\right) f=\beta_{u} v_{u}+N_{i}(E(A / P)),
$$

where $\beta_{u} \in E(A / P)$. If $u \neq t,\left(a_{u, s} e_{u, s}\right) f=0$. If $u \geqslant t$,

$$
\left(e_{i u} a_{u s} e_{u, s}\right) f=\left(a_{u s} e_{t, s}\right) f=\beta_{u} v_{i}+N_{i}(E(A / P)) .
$$

But since $a_{u, s} \in J$, we also have $\left(a_{u, s} e_{t, s}\right) f=a_{u, s} c v_{t}+N_{l}(E(A / P))$. Thus $a_{u, s} c=\beta_{u}$, and

$$
\begin{aligned}
a t+c r v_{s}+N_{\imath}( & E(A / P)) \\
& =\left(-r e_{s, s}\right) f+c r v_{s}+N_{t}(E(A / P)) \\
& =-\sum_{u \leqslant s}\left[\left(a_{u, s} e_{u, s}\right) f\right]+c \sum_{u \leqslant s}\left[a_{u, s} v_{u}+N_{b}(E(A / P)]\right. \\
& =-\sum_{u \leqslant s}\left[a_{u, s} c v_{u}+N_{t}(E(A / P))\right]+c \sum_{u \leqslant s}\left[a_{u, s} v_{u}+N_{t}(E(A / P))\right] \\
& =0 .
\end{aligned}
$$

Therefore, $h$ is well defined and properly extends $f$. This contradiction implies that $V_{P, l}$ is injective, and the proof is complete. 
Proposition 3.2. If $s, t \in T, P, Q \in \operatorname{Spec} A$, then $\operatorname{Hom}_{R}\left(V_{P, s}, V_{Q .1}\right) \neq(0)$ if and only if $s \leqslant t$ and $P \subseteq Q$.

Proof. Assume that $s \leqslant t, P \subseteq Q$. Then $N_{t}(E(A / P)) \subseteq N_{s}(E(A / P))$ and there is a surjection $\Pi: V_{P, s} \rightarrow V_{P, t}$. Since $P \subseteq Q$, there is a natural surjection $A / P \rightarrow A / Q$ which induces a nonzero $R$-map from $K_{l}(A / P)$ to $K_{l}(A / Q)$. This in turn induces a nonzero $R$-map from $V_{P, t}$ to $V_{Q, V}$. The composition of those two maps is a nonzero map in $\operatorname{Hom}_{R}\left(V_{P, s}, V_{Q, t}\right)$.

Conversely, assume that $0 \neq f \in \operatorname{Hom}_{R}\left(V_{P_{s},}, V_{Q, \ell}\right)$. Then there exists $\alpha \in A / Q$ such that $0 \neq \alpha v_{l}+N_{l}(E(A / Q))=v f$, some $v \in V_{P, s}$. Thus

$$
\left(e_{t, t} v\right) f=\left[\beta v_{t}+N_{s}(E(A / P))\right] f=\alpha v_{t}+N_{t}(E(A / Q)), \quad \beta \in E(A / P) .
$$

Since $f$ is well defined, $\beta v_{t} \notin N_{s}(E(A / P))$ and hence $s \leqslant t$. Secondly, the map $f: A\left(\beta v_{l}\right)+N_{s}(E(A / P)) \rightarrow A\left(\alpha v_{t}\right)+N_{l}(E(A / Q))$ induces a nonzero $A$-map from $E(A / P)$ to $E(A / Q)$. Thus $P \subseteq Q$. This completes the proof.

COROLLARY 3.3. Spec $R$ with the Zariski topology is homeomorphic to $(\operatorname{Spec} A) \times T$ with the product topology where Spec $A$ has the Zariski topology and $T$ has the order topology.

Proof. The result follows immediately from Propositions 3.1 and 3.2.

Note that an arbitrary open set $U$ in $\operatorname{Spec} R$ may be expressed as a union $U=U_{t \in T}(\mathcal{O}(t) \times t)$, where $\mathcal{O}(t)$ is an open subset of Spec $A$ for each $t$ and $\mathcal{O}(s) \subseteq \mathcal{O}(t)$ if $t \leqslant s$ in $T$. Let $T^{*}$ denote the set of minimal elements of $T$ and if $t \in T^{*}$, let $n_{t}$ denote the number of $s \in T$ such that $t \leqslant s$. If $U$ is an open subset of $\operatorname{Spec} R$, let $\Pi_{T}(U)$ be the set of $t \in T$ such that $\mathscr{O}(t) \neq \varnothing$ in the decomposition above.

Proposition 3.4. With notations as above, let $U=\bigcup_{t \in T}(\mathcal{O}(t) \times t)$ be an open subset of $\operatorname{Spec} R$, and let

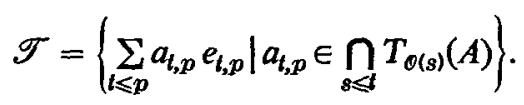

Then $T_{U}(R)=\mathscr{T}$.

Proof. Let $a \in R, a \notin \mathscr{T}$. Then some "coordinate" $a_{u, s}$ of $a$ is not in $\bigcap_{w \leqslant u} T_{O(w)}(A)$. Hence, for some $p \leqslant u, a_{u, s} \notin T_{\mathcal{O}(p)}(A)$; and there exists $P \in \mathcal{O}(p)$ such that $a_{u, s} E(A / P) \neq(0)$. But then, since $s \geqslant p, \quad 0 \neq a_{u, s} e_{u, s} \beta_{s} v_{s}+N_{p}(E(A / P)) \in V_{P, p}$ for some $\beta_{s} \in E(A / P)$. Thus $a \notin T_{U}(R)$ and $T_{U}(R) \subseteq \mathscr{T}$.

Now let $a \in R, a \notin T_{U}(R)$. There exists $\left[V_{P, s}\right] \in U, v \in V_{P_{s} s}$ such that $a v \neq 0$. Write $v=\sum_{u \triangleq s}\left[\beta_{u} v_{u}+N_{s}(E(A / P))\right]$ and choose $w \in T$ such that $a \beta_{w} v_{w}+N_{s}(E(A / P)) \neq 0$. 
Then

$$
\begin{aligned}
0 \neq a \beta_{w} v_{w}+N_{s}(E(A / P)) & =\sum_{t \leqslant w}\left[a_{t, w} e_{t, w} \beta_{w} v_{w}+N_{s}(E(A / P))\right] \\
& =\sum_{t \leqslant w}\left[a_{t, w} \beta_{w} v_{t}+N_{s}(E(A / P))\right] .
\end{aligned}
$$

Choose $t \geqslant s$ such that $a_{t, w} \beta_{w} \neq 0$. Then $a_{t, w} \notin T_{\mathcal{O}(s)}(A)$. Since $s \leqslant t$, this implies that $a \notin \mathscr{T}$. Thus $\mathscr{T}=T_{U}(R)$ and the proof is complete.

Let $S^{*}(U)=T^{*} \cap \Pi_{T}(U)$ and note that since $s \leqslant t$ implies that $T_{\mathcal{O}(s)}(A) \subseteq T_{o(t)}(A)$, $T_{U}(R)=\left\{\sum_{t \leqslant p} a_{t, p} e_{t, p}\right\}$, where the coefficients $a_{t, p}$ range over the set

$$
\bigcap\left\{T_{\mathcal{O}(s)}(A) \mid s \in S^{*}(U), s \leqslant t\right\} .
$$

Thus if $U=\bigcup\{\mathcal{O}(t) \times t \mid t \in T\}$ and $U^{*}=\bigcup\left\{\mathcal{O}(t) \times t \mid t \in S^{*}(U)\right\}$, where $U$ and $U^{*}$ are open subsets of $\operatorname{Spec} R$, then $T_{U}(R)=T_{U *}(R)$. This implies that the restriction map $\varphi_{U, U^{*}}: \boldsymbol{R}_{U \rightarrow R_{U^{*}}}$ is an injection. We first describe the rings $\boldsymbol{R}_{U^{*}}$ where $U^{*}=\bigcup_{i \in S}(\mathcal{O}(t) \times t), S \subset T^{*}$; then we describe the rings $R_{U}$ where $U$ is an arbitrary open set in $\operatorname{Spec} R$.

Proposition 3.5. If $t \in T^{*}, \mathcal{O}(t)$ is an open subset of $\operatorname{Spec} A$, and $U=\mathcal{O}(t) \times t$, then $R_{U}$ is isomorphic to the ring of $n_{t} \times n_{t}$ matrices over $A_{\mathcal{O}(t)}$. More generally, if $S \subset T^{*}$ and $U=\bigcup_{t \in S}(\mathcal{O}(t) \times t), \mathcal{O}(t)$ open in $\operatorname{Spec} A$ for each $t \in S$, then $R_{U}=\Pi_{i \in S} R_{(\mathcal{O}(t) \times l)}$.

Note: The proofs of this proposition and of the following Theorem 3.6 are quite technical. We give only outlines of the proofs.

ProOF. Let $U=\mathcal{O}(t) \times t$, as in the statement of the proposition. Let $W_{t}=\amalg_{P \in \mathcal{O}(t)} E(A / P)$. Then $V_{U}=\amalg_{P \in \mathcal{O}(t)} V_{P i} \cong V\left(W_{t}\right) / N_{t}\left(W_{t}\right)$. Let $\varphi \in E_{U}$. Then for each $s \geqslant t$ and each $u \geqslant t$ we define a map $\varphi_{s, u}: W_{l} \rightarrow W_{t}$ as follows: if $w \in W_{t}$ then $w \varphi_{s, u}$ is the $u$ th "coordinate" of $\left\{w v_{s}+N_{t}\left(W_{t}\right)\right\} \varphi$. Hence

$$
\left\{w v_{s}+N_{l}\left(W_{t}\right)\right\} \varphi=\sum_{u \geqslant t}\left(w \varphi_{s, u}\right) v_{u}+N_{\ell}\left(W_{\ell}\right) \text {. }
$$

Then $\varphi_{s, u} \in \operatorname{End}_{A}\left(W_{t}\right)$. It can be verified that $\varphi_{s, u}=0$ if $u \neq s$ and that $\varphi_{s, s}=\varphi_{l, t}$ for all $s \geqslant t$. This permits us to identify $E_{U}$ with End $_{A}\left(W_{t}\right)$ via the map $\varphi \rightarrow \varphi_{t, l}$.

Let $B=\left[b_{r, s}\right], r, s \geqslant t$, be an $n_{t} \times n_{t}$ matrix with entries in $A_{\mathcal{O}(t)}=\operatorname{Biend}_{A}\left(W_{t}\right)$. Define a map $q_{B}: V_{U} \rightarrow V_{U}$ by

$$
q_{B}: w v_{s}+N_{t}\left(W_{l}\right) \mapsto \sum_{r \geqslant l}\left(\left(b_{r, s} w\right) v_{r}+N_{t}\left(W_{t}\right)\right), \quad \text { all } s \geqslant t .
$$

Then one can check that $q_{B} \in R_{U}$. Conversely, if $q \in R_{U}, w \in W_{t}$, we let $b_{r, s}(w)$ denote the $r$ th "coordinate" of $q\left\{w v_{s}+N_{i}\left(W_{t}\right)\right\}$. Then it can be shown that $b_{r, s}$ can be chosen independent of $w$, and $b_{r, s} \in A_{\mathcal{O}(t)}$, for all $r, s \geqslant t$. Thus we obtain an isomorphism between $R_{U}$ and the ring of $n_{t} \times n_{t}$ matrices over $A_{O(t)}$. 
Now suppose that $S \subset T^{*}, U=\bigcup_{i \in S}(\mathcal{O}(t) \times t)$, where $\mathcal{O}(t)$ is an open subset of $\operatorname{Spec} A$ for each $t \in S$. Proposition 3.2 implies that if $s, t \in S, s \neq t$, then there are no nonzero $R$-maps between $V_{(\mathcal{O}(t) \times t)}$ and $V_{(O(s) \times s)}$. Hence

$$
\operatorname{End}_{R} V_{U}=\prod_{i \in s} \operatorname{End}_{R} V_{(\theta(t) \times(t)},
$$

which implies that Biend $_{R} V_{U}=\prod_{t \in S}$ Biend $_{R}\left(V_{(O(t) \times t)}\right)=\prod_{t_{\epsilon} s} R_{(O(t) \times t)}$. This concludes the proof.

We now turn to the computation of $R_{U}$, where $U=\bigcup_{t \in T}(\theta(t) \times t)$ is an arbitrary open subset of $\operatorname{Spec} R$. If $S^{*}(U)$ and $U^{*}$ are defined as above, then we must characterize those elements $\left(q_{p}\right) \in \prod_{p \in S^{*}(U)} \operatorname{Biend}_{R}\left(V_{(\mathcal{O}(p) \times p)}\right)$ such that $\left(q_{p}\right)$ is the restriction to $V_{U}$, of an element of Biend $V_{R} V_{U}$. As in the proof of Proposition 3.5 , we let $W_{p}=\amalg_{P \in \mathcal{O}(p)} E(A / P)$ and note that $V_{(\mathcal{O}(p) \times p)}=V\left(W_{p}\right) / N_{p}\left(W_{p}\right)$. By Proposition 3.5, we may associate to each biendomorphism $q_{p}$ of $V_{O(p) \times p}$ an $n_{p} \times n_{p}$ matrix $\left[b(p)_{s, l}\right]$ with entries in $A_{\mathscr{O}(p)}$ such that

$$
q_{p}\left[w v_{t}+N_{p}\left(W_{p}\right)\right]=\sum_{s \geqslant p}\left[\left(b(p)_{s, t} w\right) v_{s}+N_{p}\left(W_{p}\right)\right], \quad t \geqslant p .
$$

THEOREM 3.6. With notations as above, the ring $R_{U}$ of sections over $U$ may be identified with the subring of $\Pi_{p \in s^{*}(U)} \operatorname{Biend}_{R}\left(V_{(\mathcal{O}(p) \times p)}\right)$ consisting of those elements $\left(q_{p}\right)$ such that the associated matrices $\left[b(p)_{s, t}\right]$ satisfy the following two conditions:

(1) For each $p \in S^{*}(U)$ and $s, t \geqslant p$ such that there exists $k \in \Pi_{T}(U)$ with $p \leqslant k \leqslant s$ but $k \leqslant t$, it is true that

$$
b(p)_{s, t} \in \bigcap\left\{T_{\mathscr{O}(r)}\left(A_{\mathcal{O}(p)}\right) \mid r \in \Pi_{T}(U), p \leqslant r \leqslant s, r \nless t\right\} .
$$

(2) If $p, m \in S^{*}(U)$ and $s, t \in T$ such that $s, t \geqslant p, s, t \geqslant m$, and if there exists $k \in \prod_{T}(U)$ such that $s, t \geqslant k$ and $k \geqslant p, m$, then

$$
\rho_{\mathcal{O}(p), \mathcal{O}(k)}\left(b(p)_{s, t)}\right)=\rho_{\mathcal{O}(m), \mathcal{O}(k)}\left(b(m)_{s, t}\right),
$$

where $\rho_{\mathcal{O}(p), \mathcal{O}(k)}$ and $\rho_{\mathcal{O}(m), \mathcal{O}(k)}$ are the restriction maps from $A_{\mathcal{O}(p)}$ to $A_{\mathcal{O}(k)}$ and from $A_{\mathcal{O}(m)}$ to $A_{\mathcal{O}(k)}$, respectively.

Proof. For $s, t \in T, s \leqslant t$, let $\theta_{s, t}: W_{s} \rightarrow W_{t}$ be the natural projection; and define $F_{s i}: V\left(W_{\mathrm{s}}\right) / N_{\mathrm{s}}\left(W_{\mathrm{s}}\right) \rightarrow V\left(W_{t}\right) / N_{\mathrm{l}}\left(W_{t}\right)$ by $F_{\mathrm{s}, t}: w v_{u}+N_{s}\left(W_{\mathrm{s}}\right) \mapsto\left(w \theta_{\mathrm{s}, t}\right) v_{u}+N_{t}\left(W_{t}\right)$ for all $u \geqslant s$. For $q \in R_{U}$ let $q_{p}$ be the restriction of $q$ to $V\left(W_{p}\right) / N_{p}\left(W_{p}\right)$, and let $\left[b(p)_{s, t}\right]$ be the matrix associated with $q_{p}, p \in S^{*}(U)$. Let $p \in S^{*}(U), s, t \geqslant p$ and $k \in \Pi_{T}(U)$ such that $p \leqslant k \leqslant s, s \leqslant t$. Then one can show that $\left\{q_{p}\left(w v_{l}+N_{p}\left(W_{p}\right)\right)\right\} F_{p, k}=0$. From this one can deduce that $b(p)_{s, l} \in T_{\mathscr{O}(k)}\left(A_{\mathscr{C}(p)}\right)$.

Now suppose that $p, m, s, t, k$ are as in (2). Then

$$
\left.q_{k}\left[w v_{l}+N_{k}\left(W_{k}\right)\right]=\sum_{l \geqslant k}\left[\left(b(p)_{l, l} w\right) v_{l}+N_{l}\left(W_{l}\right)\right]=\sum_{l \geqslant k}\left[b(m)_{l, l} w\right) v_{l}+N_{l}\left(W_{l}\right)\right]
$$


So $\rho_{\mathscr{O}(p), \mathcal{O}(k)}\left(b(p)_{l, \ell}\right)=\rho_{\mathcal{O}(m), \mathcal{O}(k)}\left(b(p)_{l, l}\right)$, for all $l \geqslant k$. Hence

$$
\rho_{\mathcal{O}(p), \mathcal{O}(k)}\left(b(p)_{s, t}\right)=\rho_{\mathcal{O}(m) \mathcal{O}(k)}\left(b(m)_{s, t}\right) \text {. }
$$

Now assume that $\left(q_{p}\right) \in \Pi_{p_{\in S^{*}(U)}} \operatorname{Biend}_{R}\left(V\left(W_{p}\right) / N_{p}\left(W_{p}\right)\right)$ and assume that the matrices $\left[b(p)_{s t}\right]$ associated with the $q_{p}$ satisfy (1) and (2). For every $t \in \Pi_{T}(U)$ choose $p \in S^{*}(U)$ such that $p \leqslant t$, and define $q_{t} \in \operatorname{Biend}_{R}\left(V\left(W_{t}\right) / N_{l}\left(W_{t}\right)\right)$ by

$$
q_{t}\left[w v_{s}+N_{l}\left(W_{t}\right)\right]=\sum_{k \geqslant l}\left[\left(b(p)_{k, s} w\right) v_{s}+N_{t}\left(W_{t}\right)\right]
$$

where $s \geqslant t$. Then $q_{t}$ is well defined independent of $p$ by (1) and (2). Now $\left\{q_{t} \mid t \in \Pi_{T}(U)\right\}=q$ defines an $A$-linear map of $V_{U}$ to itself, and the restriction of $q$ to $V\left(W_{p}\right) / N_{p}\left(W_{p}\right), p \in S^{*}(U)$, is $q_{p}$. It can be verified that, for $r, w \in \Pi_{T}(U), r \leqslant w$, one has

$$
\left.\left\{q_{r}\left(w_{l} v_{l}+N_{r}\left(W_{r}\right)\right)\right\} \varphi_{r, w}=q_{w}\left\{\left(w_{l} v_{l}+N_{r}\left(W_{r}\right)\right)\right\} \varphi_{r, w}\right\} .
$$

From this one deduces that $q \in \operatorname{Biend}_{R}\left(V_{U}\right)$. This completes the proof.

\section{Examples}

(1a) Let $T$ be a linearly ordered set of $n$ elements, $T=\{1,2, \ldots, n\}, R$ the incidence algebra of $T$ over a field $k$. Then $R$ is the algebra of $n \times n$ upper triangular matrices over $k$. An open set $U$ is of the form $U(i)=\{1,2, \ldots, i\}, 1 \leqslant i \leqslant n$. Then $R_{U(n)}=R$, and $R_{u(1)}$ is the full ring of $n \times n$ matrices over $k$. Let $1<i<n$. The relation $\sigma$ on $T$ defined by $U(i)$ (see Section 2) is such that $j \sigma k$ whenever $j \leqslant k$ and also whenever $k \geqslant i$. Thus $R_{U(i)}$ can be identified with the ring of all $n \times n$ matrices $\left[\alpha_{j, k}\right]$ such that $\alpha_{j, k}=0$ if $k<i$ and $k<j$.

(1b) Let $T$ be as above, $R$ the incidence algebra of $T$ over a commutative noetherian ring $A$. Then $R$ is the algebra of $n \times n$ upper triangular matrices over $A$. An open set $U$ in $\operatorname{Spec} R$ is of the form $U=\bigcup_{t \leqslant i}(\mathcal{O}(t) \times t)$, where $\varnothing \neq \mathcal{O}(t) \subseteq \mathcal{O}(s)$ if $t \geqslant s$. If $i=1, R_{U}$ is the full ring of $n \times n$ matrices over $A_{\mathcal{O}(1)}$. If $i>1, R_{U}$ is the ring of $n \times n$ matrices over $A_{\mathscr{O}(1)}$ of the form $\left[\alpha_{j, k}\right]$ where $\alpha_{j, k} \in T_{\mathscr{O}(k+1)}\left(A_{\mathscr{O}(1)}\right)$ if $k<j$ and $k<i$.

(2) Let $T$ be the linearly ordered set $\{1,2,3\}, A=k[x, y] /(x y)$, where $k$ is a field, $x$ and $y$ are indeterminates. Let $R$ be the incidence algebra of $T$ over $A$, and let $U$ be the open set $U=(\operatorname{Spec} A \times\{1\}) \cup(\mathcal{O}(y) \times\{2\})$, where $\mathcal{O}(y)=\{P \in \operatorname{Spec} A \mid y \notin P\}$. Then by (1b) above, $R_{U}$ is the ring of $3 \times 3$ matrices over $A$ of the form

$$
\left[\begin{array}{ccc}
a_{11} & a_{12} & a_{13} \\
a_{21} x & a_{22} & a_{23} \\
a_{31} x & a_{32} & a_{33}
\end{array}\right] .
$$

(3) If $A$ is an integral domain and $\varnothing \neq \mathcal{O} \subseteq \mathcal{O}^{\prime}$ are open subsets of $\operatorname{Spec} A$, then $T_{0}\left(A_{0^{\circ}}\right)=(0)$. Thus condition (1) of Theorem 3.6 reduces to: $\left(1^{*}\right)$ For each 
$y \in S^{*}(U)$ and $s, t \geqslant y$ such that there exists $s^{\prime} \in \Pi_{T}(U)$ with $y \leqslant s^{\prime} \leqslant s$, but $s^{\prime} \leqslant t$, $b(y)_{s, t}=0$.

Hence if $T=\{1,2,3,4,5\}$ is the partially ordered set defined by: $1 \leqslant 3 \leqslant 5$, $2 \leqslant 4 \leqslant 5,1 \leqslant 4,2 \leqslant 3, A=\mathbf{Z}$, and $R$ is the incidence algebra of $T$ over $\mathbf{Z}$, then $R$ is the ring of $5 \times 5$ matrices over $\mathbf{Z}$ of the form

$$
\left[\begin{array}{ccccc}
z_{11} & 0 & z_{13} & z_{14} & z_{15} \\
0 & z_{22} & z_{23} & z_{24} & z_{25} \\
0 & 0 & z_{33} & 0 & z_{35} \\
0 & 0 & 0 & z_{44} & z_{45} \\
0 & 0 & 0 & 0 & z_{55}
\end{array}\right] .
$$

Let $U=(\mathscr{O}(2) \times\{1\}) \cup(\mathcal{O}(3) \times\{2\}) \cup(\mathcal{O}(6) \times\{4\})$, where $\mathscr{O}(i)=\{P \in \mathrm{Spec} \mid i \notin P\}$. Then $R_{U}$ is the set of ordered pairs of the form

$$
\left[\begin{array}{cccc}
\alpha_{11} & \alpha_{13} & \alpha_{14} & \alpha_{15} \\
0 & \alpha_{33} & \alpha_{34} & \alpha_{35} \\
0 & 0 & \alpha_{44} & \alpha_{45} \\
0 & 0 & \alpha_{54} & \alpha_{55}
\end{array}\right],\left[\begin{array}{cccc}
\beta_{22} & \beta_{23} & \beta_{24} & \beta_{25} \\
0 & \beta_{33} & \beta_{34} & \beta_{35} \\
0 & 0 & \beta_{44} & \beta_{45} \\
0 & 0 & \beta_{54} & \beta_{55}
\end{array}\right]
$$

with each $\alpha_{j, k} \in \mathbf{Z}_{\mathscr{O}(2)}$, each $\beta_{s, i} \in \mathbf{Z}_{\mathcal{O}(3)}$, and the restrictions of $\alpha_{44}, \alpha_{45}, \alpha_{54}, \alpha_{55}$ to $\mathbf{Z}_{\mathscr{O}(8)}$ agree with the restrictions of $\beta_{44}, \beta_{45}, \beta_{54}, \beta_{55}$ to $Z_{\mathcal{G}(6)}$, respectively.

If $A$ is a field $k, T$ the partially ordered set above, $R$ the incidence algebra of $T$ over $k$ and $U$ the open set $\{1,2,4\}$; then $R_{U}$ is the set of ordered pairs of matrices over $k$ of the form

$$
\left[\begin{array}{cccc}
\alpha_{11} & \alpha_{13} & \alpha_{14} & \alpha_{15} \\
0 & \alpha_{33} & \alpha_{34} & \alpha_{35} \\
0 & 0 & \alpha_{44} & \alpha_{45} \\
0 & 0 & \alpha_{54} & \alpha_{55}
\end{array}\right],\left[\begin{array}{cccc}
\beta_{22} & \beta_{23} & \beta_{24} & \beta_{25} \\
0 & \beta_{33} & \beta_{34} & \beta_{35} \\
0 & 0 & \alpha_{44} & \alpha_{45} \\
0 & 0 & \alpha_{54} & \alpha_{55}
\end{array}\right]
$$

(4) Let $T=\{1,2,3,4,5\}$ be the partially ordered set defined by: $1 \leqslant 3 \leqslant 5$, $2 \leqslant 4 \leqslant 5,1 \leqslant 4$ and $A=k[x, y, z] /(x y z)$, where $k$ is a field and $x, y$ and $z$ are indeterminates. Let $R$ be the incidence algebra of $T$ over $A, U$ the open set $(\mathscr{O}(x) \times\{1\}) \cup(\mathcal{O}(z) \times\{2\}) \cup(\mathcal{O}(x y) \times\{3\}) \cup(\mathcal{O}(x z) \times\{4\})$, where $\mathcal{O}(a)=\{P \in \operatorname{Spec} A \mid a \notin P\}$. Then $R_{U}$ is the set of ordered paris of matrices of the form

$$
\left[\begin{array}{cccc}
\alpha_{11} & \alpha_{13} & \alpha_{14} & \alpha_{15} \\
\alpha_{31} z & \alpha_{33} & \alpha_{34} z & \alpha_{35} \\
\alpha_{41} y & \alpha_{43} y & \alpha_{44} & \alpha_{45} \\
0 & \alpha_{53} y & \alpha_{54} z & \alpha_{55}
\end{array}\right],\left[\begin{array}{ccc}
\beta_{22} & \beta_{24} & \beta_{25} \\
\beta_{42} y & \beta_{44} & \beta_{45} \\
\beta_{52} y & \beta_{54} & \beta_{55}
\end{array}\right]
$$


such that each $\alpha_{j, k} \in A_{x}$, each $\beta_{s, t} \in A_{z}$, and such that the restrictions of $\alpha_{44}, \alpha_{45}$, $\alpha_{54}, \alpha_{55}$ to $A_{x z}$ agree respectively with the restrictions of $\beta_{44}, \beta_{45}, \beta_{54}, \beta_{55}$ to $A_{x z}$ ( $A_{a}$ is used to denote $A_{\mathscr{O}(a)}$.)

\section{References}

B. Goldston and A. C. Mewborn (1975), "A structure sheaf for a non-commutative noetherian ring", Bull. Amer. Math. Soc. 81, 944-946.

B. Goldston and A. C. Mewborn (1977), "A structure sheaf for a noncommutative noetherian ring", J. Algebra 47, 18-28.

R. Gordon and J. C. Robson (1973), Krull Dimension (Mem. Amer. Math. Soc. 133, Providence, R.I., U.S.A.)

B. T. Stenström (1971), Rings and Modules of Quotients (Lecture Notes in Mathematics 237, Springer-Verlag, Berlin).

Randolph Macon Womans College

Lynchburg, Virginia, USA
University of North Carolina

Chapel Hill, North Carolina, USA 